\title{
Optical analysis of textured plastic substrates to be used in thin silicon solar cells
}

\author{
J. Escarré, F. Villar, M. Fonrodona, D. Soler, J.M. Asensi, J. Bertomeu, J. Andreu \\ CeRMAE - Dept. Física Aplicada i Òptica, Universitat de Barcelona, Av. Diagonal 647, 08028 Barcelona, SPAIN
}

Light confinement strategies in thin film silicon solar cells play a crucial role in the performance of the devices. In this work, the possible use of Ag coated stamped polymers as reflectors to be used in n-i-p solar cells is studied. Different random roughnesses (nanometer and micrometer size) have been transferred on poly(methylmethacrylate) (PMMA) by hot embossing. Morphological and optical analyses of masters, stamped polymers and reflectors have been carried out evidencing a positive surface transference on the polymer and the viability of a further application in solar cells.

Keywords: Hot embossing; Light trapping; PMMA; Random texture; Solar Cell

\section{Introduction}

There is a growing interest in the development of thin film silicon solar cells on flexible plastic substrates, as they allow cost reduction in the production process by using roll-to-roll deposition systems and facilitate monolithic interconnection of the cells to produce a photovoltaic module [1]. Plastic substrates are also used in organic solar cells.

Amorphous (a-Si:H) and microcrystalline ( $\mu \mathrm{c}-\mathrm{Si}: \mathrm{H})$ silicon photovoltaic devices rely on the electric field to collect the photogenerated carriers. In those devices, the active layer is intrinsic and the electric field and the resulting device parameters highly depend on its thickness. If this thickness is optimised to ensure good electrical collection, the layer is not optically dense enough to absorb light at long wavelengths. In this conditions, the short-circuit current critically depends on optical strategies to enhance the light absorption in the active layer. 
The standard strategy in light confinement is to use Transparent Conductive Oxides (TCO) with controlled roughness as front contacts [2] and the development of back reflectors based on TCO and metal (Ag or $\mathrm{Al}$ ) layers [3]. The first approach requires the use of thick TCO layers, which are difficult to deposit on top of plastic substrates as stress arising from TCO deposition can affect the polymer. An alternative approach would be to transfer the controlled roughness to the plastic substrate and deposit the metal/TCO/n-i-p/TCO structure on top of this textured plastic surface. This approach has been yet tested on glass substrates [4].

Hot embossing [5] and nanoimprinting lithography (NIL) [6] are imprint techniques used to transfer superficial features on a polymer surface. These techniques allow the reproduction of superficial structures with sizes ranging from millimeters to nanometers. The stamping of regular structures on poly(methylmethacrylate) (PMMA) is the most commonly used application of these imprinting processes. After fabrication, these surfaces can be used in different applications like the fabrication of fluidic devices [7] or as support materials for biomedical experimentation [8].

In the present work we present a preliminary study to analyse the viability of the use of polymer substrates on which a random texture has been transferred by means of hot embossing processes. In these first steps, we used PMMA, knowing that this polymer is not the ideal candidate to be used as substrate in thin silicon solar cells due to its low temperature resistance, which makes it difficult to deposit good quality silicon on top. Nevertheless, PMMA is commonly used in embossing processes to reproduce regular structures, what makes it a good candidate for a preliminary study of random roughness transference. Materials exhibiting clear difference in the size of the roughness (nanometer vs. micrometer) have been used as masters to reproduce their surface on top of the PMMA. In order to finish the back reflector structure, an Ag layer was deposited on top of the textured PMMA. The optical analysis has been carried out in an experimental set-up which allowed the measurement of the angular distribution function $(\mathrm{ADF})$ of the light scattered by a rough surface. This system is used to test the optical behaviour of the back reflector structures obtained on PMMA.

\section{Experimental}


The polymer used has been the PMMA ( 0.1 mm-thick). Its glass transition temperature is around $105^{\circ} \mathrm{C}$ and its maximum working temperature, $130^{\circ} \mathrm{C}$. The imprinting of the PMMA has been done using a Jenoptik HEX 01 hot embossing system (Jenoptik Mikrotechnik GmbH, Germany). In this system, a master, the surface of which is to be transferred, is put on the PMMA. Next, the entirety is heated above the polymer glass temperature $\left(\mathrm{T}=120^{\circ} \mathrm{C}\right.$ in our case), what causes the PMMA to soften, making it able to adopt the master surface when a force is applied to PMMA-master set during a set time ( $\mathrm{F}=400 \mathrm{~N}$ and $\mathrm{t}=600 \mathrm{~s}$ in this study). Finally, the PMMA has to be cooled below its glass temperature before the master can be carefully released. The observable features transferred on the polymer surface are, thus, the negative of the texture of the master. The temperature at which the PMMA is heated during the stamping, the applied force and the time during which the force is applied are the main parameters to control the stamping process.

Masters used for the hot embossing were commonly available frosted glass, where the surface had been etched using HF to produce the frosted appearance, and commercially available Asahi-U TCO (Asahi Glass Co., Japan). Both masters exhibited random surface structures with roughness RMS values in the order of microns in the case of the frosted glass and of tens of nanometers for the Asahi-U. Finally, the reflector structure was finished by evaporating a thin $\mathrm{Ag}$ layer $(185 \mathrm{~nm})$ on top of the textured polymer substrates. Thermal evaporation was carried out in an Edwards E306A system at room temperature.

Morphological analysis of masters, imprinted polymers and reflectors was performed by means of scanning electronic microscopy (SEM, Strata DB235, FEI Co., USA), atomic force microscopy (AFM, Dimension 3100, Digital instruments, USA) and white light interferometry (Wyko NT110, Veeco Metrology, USA). The optical analysis was carried out in a system that allows us to measure the ADF of the light scattered by a rough surface. In figure 1, a scheme of the experimental set-up used to measure the ADF is shown. A red diode laser $(\lambda=633 \mathrm{~nm})$ was used as light source, which is guided inside the system by an optical fibre. In its way, the laser light is chopped and split in two beams to correct any possible variations in the light intensity. In the system, the light source is fixed whereas the sample can rotate around its own axis to change the incident angle and the detector rotates around the sample at a known distance. The reference used to measure the angles is also plotted in figure 1. The movements are 
controlled by step-by-step motors, corresponding one step to $0.9^{\circ}$. Light is detected by silicon detectors $\left(\right.$ area $\left.=16 \mathrm{~mm}^{2}\right)$ and the two signals (sample and reference) are measured by two lock-in amplifiers (Standford Research Systems, SR850 DSP). Using this configuration, the ADF is defined as the ratio between the energy measured per solid angle unit $\left(\mathrm{E} / \Omega_{\mathrm{det}}\right)$ and the total energy $\left(\mathrm{E}_{\mathrm{tot}}\right)$. For the random structures analysed, a spherical symmetry around the axis determined by the incident beam is assumed. This symmetry allowed us to determine all the scattered light measuring in a circle around the sample only and, thus, to calculate the transmission and reflection haze parameters.

\section{Results}

First of all, a morphological study of the two different masters used was performed. Asahi-U surface was studied using AFM and SEM whereas in the case of the frosted glass, due to its larger roughness, white light interferometry was used instead of AFM. The images obtained are shown in figure 2 . The roughness RMS values measured were $36.1 \mathrm{~nm}$ for the Asahi-U glass and $2640 \mathrm{~nm}$ for the frosted glass. A summary of the roughness RMS values measured in all the cases analysed in this study can be seen in table 1 .

After the hot embossing process of PMMA using both masters, the morphology of the stamped polymers was analysed using the same characterisation techniques employed in the characterisation of the corresponding master. The roughness RMS values obtained were $41.2 \mathrm{~nm}$ using Asahi-U as master and $2680 \mathrm{~nm}$ when having used the frosted glass. No noticeable difference was appreciated when comparing these values with the ones of the masters. Therefore, it could be concluded that a good surface transference had been obtained in the stamping process. The images of the morphological characterisation of stamped PMMA can be seen in figure 3.

As the ultimate goal of this work is the future use of textured polymers as back reflector structures in solar cells, an Ag layer was evaporated on top of the stamped polymers. Since the sample stamped with Asahi-U exhibited a smaller roughness size and it could be more easily influenced by a metallic deposition on top of it, the texture of PMMA before and after the deposition of the Ag layer were analysed to discard any softening of the texture. Figure 4 shows the AFM measurements of both samples. 
No significant influence of this layer on the initial texture was observed, as can be seen in the RMS values presented in table 1.

Next step was to optically characterise masters and reflectors by measuring the transmitted and reflected scattered light. Firstly, analyses of the masters were done. The angular distribution functions in both cases studied are shown in figure 5. The transmission (reflection) haze parameter, $H_{T}\left(H_{R}\right)$ is defined as the ratio between the transmitted (reflected) scattered light and the total transmitted (reflected) light. $\mathrm{H}_{\mathrm{T}}=$ 0.06 and $H_{R}=0.21$ were measured for Asahi-U whereas $H_{T}=0.99$ and $H_{R}=0.99$ were obtained for the frosted glass. Finally, comparison between the two PMMA reflectors was done in order to decide what kind of surface could be more interesting to use from the optical point of view. The reflected ADF in both cases (having used Asahi-U and frosted glass as masters) is represented in figure 6. Measured reflection haze parameters for the reflectors were $H_{R}=0.26$ for polymer stamped with Asahi-U and $H_{R}=0.99$ after having used the frosted glass as master.

\section{Discussion}

A comparison between the images of the PMMA surface after the embossing process (fig. 3) and those of the masters used in the process (fig. 2) exhibited similar aspects, and no significant difference was observed in the roughness RMS values between master and stamped polymer either having used Asahi-U (roughness of tens of nanometers) or frosted glass (micrometer-dimension roughness), taking into account that the latter is the negative of the former. These results indicated the viability to use hot embossing as imprinting technique to reproduce a random texture on the PMMA surface, regardless the dimensions of the master used. Additionally, the further deposition of an Ag layer on top of the textured polymer in order to build a reflector structure did not affect the final roughness of the entirety. Therefore, we could conclude that back reflectors consisting in textured polymers with a metallic coating are morphologically suitable to be used in solar cells with structure reflector/TCO/n-i-p/TCO.

It is common to use textured TCOs like Asahi-U as front contacts to enlarge the optical path, and therefore the light absorption, in solar cells. Nevertheless, the study of the light scattered by a 
commercially available Asahi substrate $\left(\mathrm{H}_{\mathrm{T}}=0.06\right)$ seemed to point to results far from the ideal case expected, in which the best confinement would be expected $\left(\mathrm{H}_{\mathrm{T}}\right.$ close to one $)$. On the contrary, the frosted glass exhibited an optical behaviour much closer to the ideal diffuser, with a measured $\mathrm{H}_{\mathrm{T}}$ of 0.99 . Therefore, frosted glass had the optical properties desired for a solar cell substrate and this fact is related with the roughness of its surface, as seen from the Haze values measured.

In figure 6, the reflected $\mathrm{ADF}$ of both reflector structures was presented. As expected, and in agreement with the results obtained for the masters, more scattered light was measured for the reflector made after the frosted glass embossed polymer. The fact that a small fraction of transmitted light was measured in these reflectors indicated that next studies must be done with thicker Ag layers. The eventual influence of this thicker Ag layers on the roughness should also be analysed. Optical results also pointed to the need to focus future work in the use of frosted glass-like textures in our solar cells.

All results presented up to now indicate the feasibility to use stamped polymers as substrates for solar cells, as we have demonstrated the viability to use imprinting techniques to reproduce random roughnesses on PMMA. Further work will deal with the use of different polymers, resistant to higher working temperatures and, thus, compatible with our device-quality silicon films.

\section{Conclusions}

Hot embossing has been proven as a viable technique to reproduce random texture on the surface of poly(methylmethacrylate) (PMMA), regardless roughness size. Besides, the subsequent deposition of an Ag layer on top of the stamped polymer did not affect the final roughness of the whole. Therefore, the back reflector structure consisting in a textured polymer plus a metallic layer on top was morphologically suitable to be used in solar cells.

Finally, optical analysis pointed that roughnesses in the order of the micron (like that of the frosted glass) showed more promising results taking into account what is desired in light confinement of solar cells. 


\section{Acknowledgements}

This work has been financed by the Spanish Government (MAT2001-3541-C03-01). The authors also want to thank Dr. Chris Mills and Dr. Elena Martínez from the Laboratori de Recerca en Nanobioenginyeria del Parc Científic de Barcelona for the stamping of the polymers and the morphological characterisation.

\section{References}

[1] M. Izu, T. Ellison, Sol. Energy Mater. Sol. Cells 78 (2003) 613

[2] J. Meier, J. Spitznagel, S. Faÿ, C. Bucher, U. Graf, U. Kroll, S. Dubail, A. Shah, Proc. of the $29^{\text {th }}$ IEEE PVSC (2002) 1118

[3] S.S. Hegedus, W.A. Buchanan, E. Eser, Proc. of $26^{\text {th }}$ IEEE PVSC (1997)

[4] N. Chuansuwanich, P.I. Widenborg, P. Campbell, A.G. Aberle, Proc. of $14^{\text {th }}$ PVSEC (2004) 325

[5] M. Hekele, W.K. Schomberg, J. Micromech. Microeng. 14 (2004) R1

[6] C.M. Sotomayor-Torres, S. Zankovych, J. Seekamp, A.P. Kam, C. Clavijo Cedeño, T. Hoffmann, J. Ahopelto, F. Reuther, K. Pfeiffer, G. Bleidiessel, G. Gruetzner, M.V. Maximov, B. Heidari, Mater. Sci. Eng. C 23 (2003) 23

[7] H. Becker, L.E. Locascio, Talanta 56 (2002) 267

[8] B. Kasemo, Surf. Sci. 500 (2002) 656 


\section{Table captions}

Table 1. Summary of the roughness RMS values for masters and stamped PMMA.

Figure captions

Figure 1. Scheme of the optical system used to measure the angular distribution function (ADF) of the light scattered by a rough surface. The reference used to measure the angles is also included.

Figure 2. SEM and AFM images of Asahi-U surface (a) and SEM and white light interferometry measurements of the surface of the frosted glass (b).

Figure 3. Textured PMMA surface images: SEM and AFM pictures having used Asahi-U as master (a) and SEM and white light interferometry pictures having used frosted glass as master (b).

Figure 4. AFM images of textured PMMA (Asahi-U master) before (a) and after (b) the deposition of a 185 nm-thick Ag layer.

Figure 5. Angular distribution function (ADF) of masters (Asahi-U and frosted glass). The ADF is defined as the ratio between the energy measured per solid angle unit $\left(\mathrm{E} / \Omega_{\mathrm{det}}\right)$ and the total energy $\left(\mathrm{E}_{\mathrm{tot}}\right)$

Figure 6. ADF of reflector structure: stamped polymer + Ag layer for the two roughnesses considered. 


\begin{tabular}{|l|c|c|}
\cline { 3 - 3 } \multicolumn{2}{|c|}{} & Roughness RMS value $(\mathrm{nm})$ \\
\hline \multirow{3}{*}{ Asahi-U } & Master & 36.1 \\
\cline { 2 - 3 } & PMMA & 41.2 \\
\cline { 2 - 3 } & PMMA-Ag(185nm) & 40.7 \\
\hline \multirow{3}{*}{ Frosted glass } & Master & 2640 \\
\cline { 2 - 3 } & PMMA & 2680 \\
\cline { 2 - 3 } & PMMA-Ag(185nm) & * not measured \\
\cline { 2 - 3 }
\end{tabular}

Table 1 


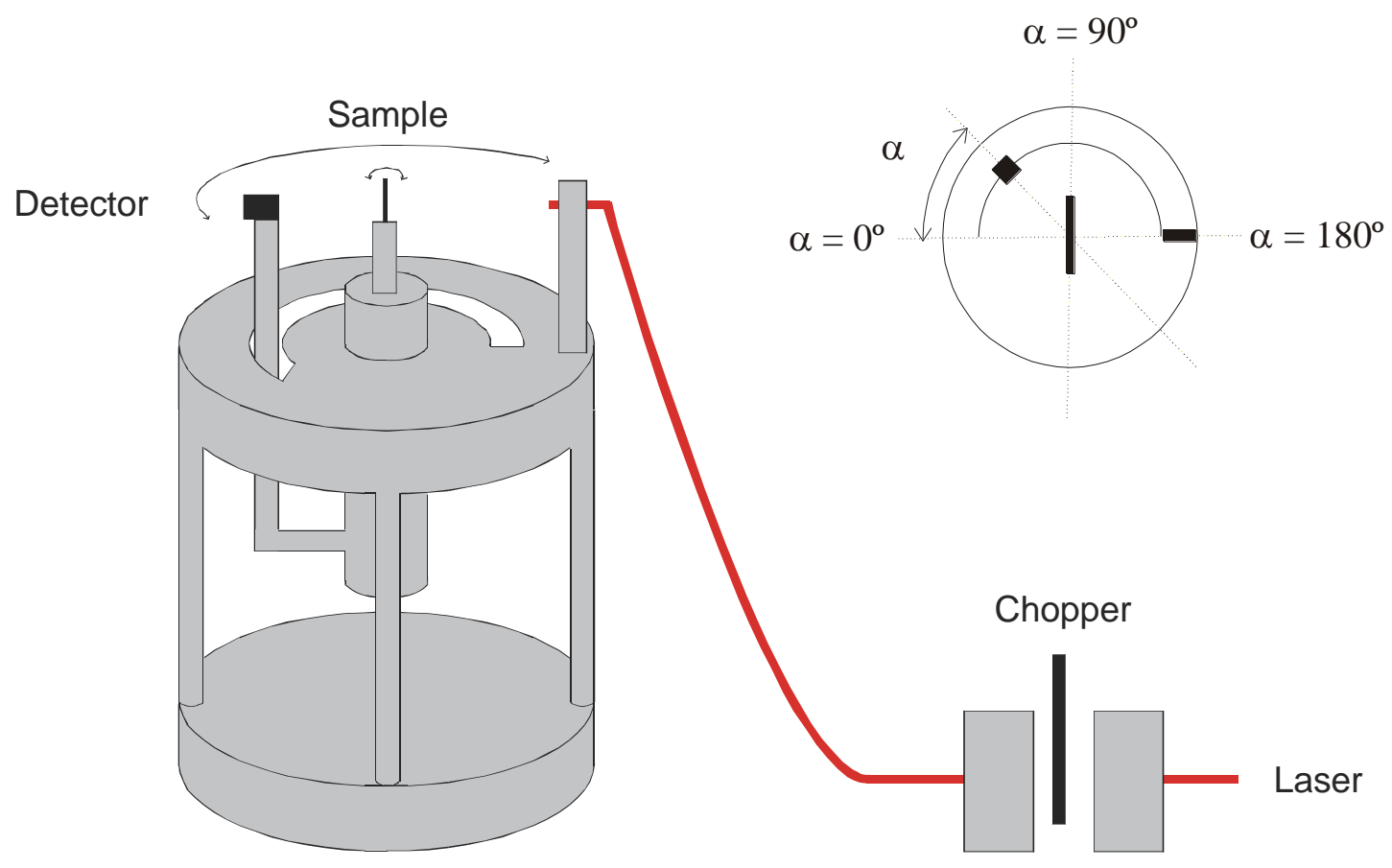

Figure 1 

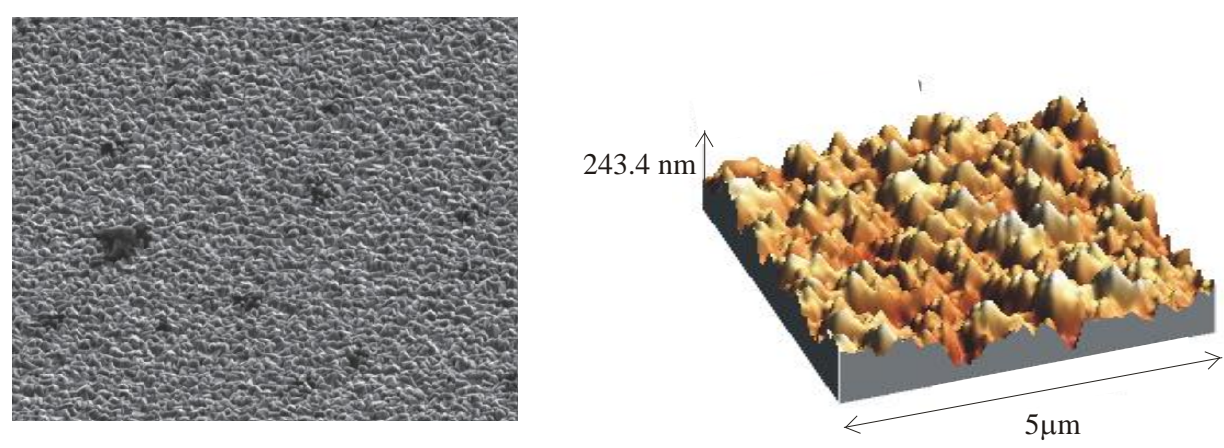

a)
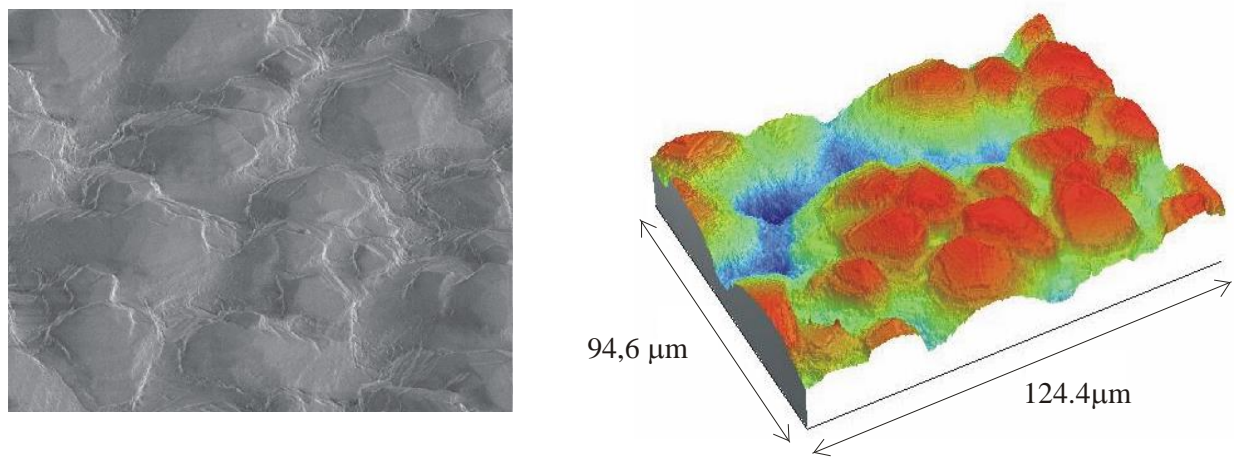

b)

Figure 2 

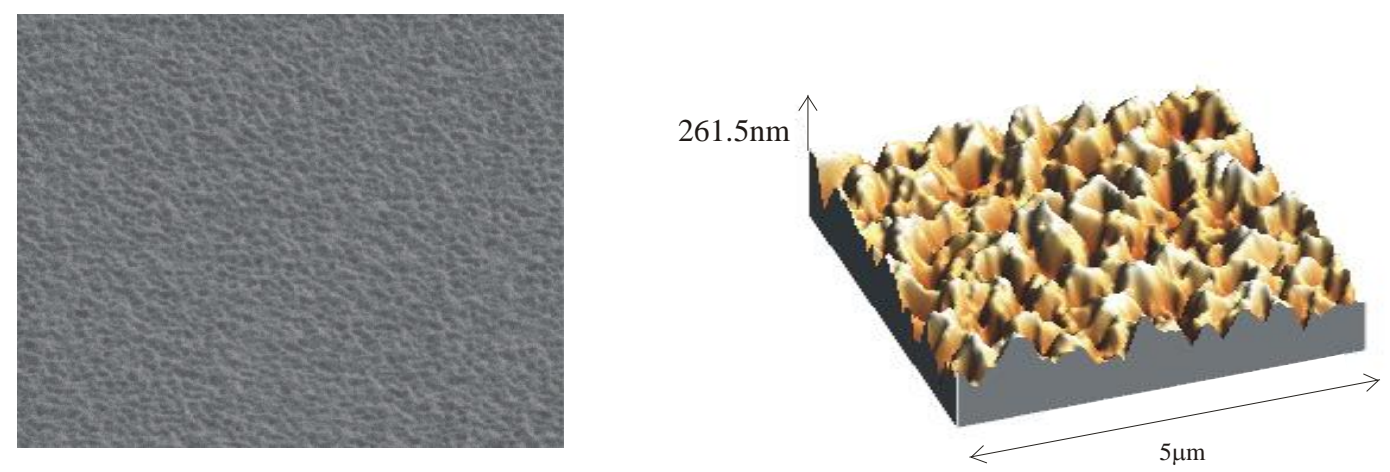

a)
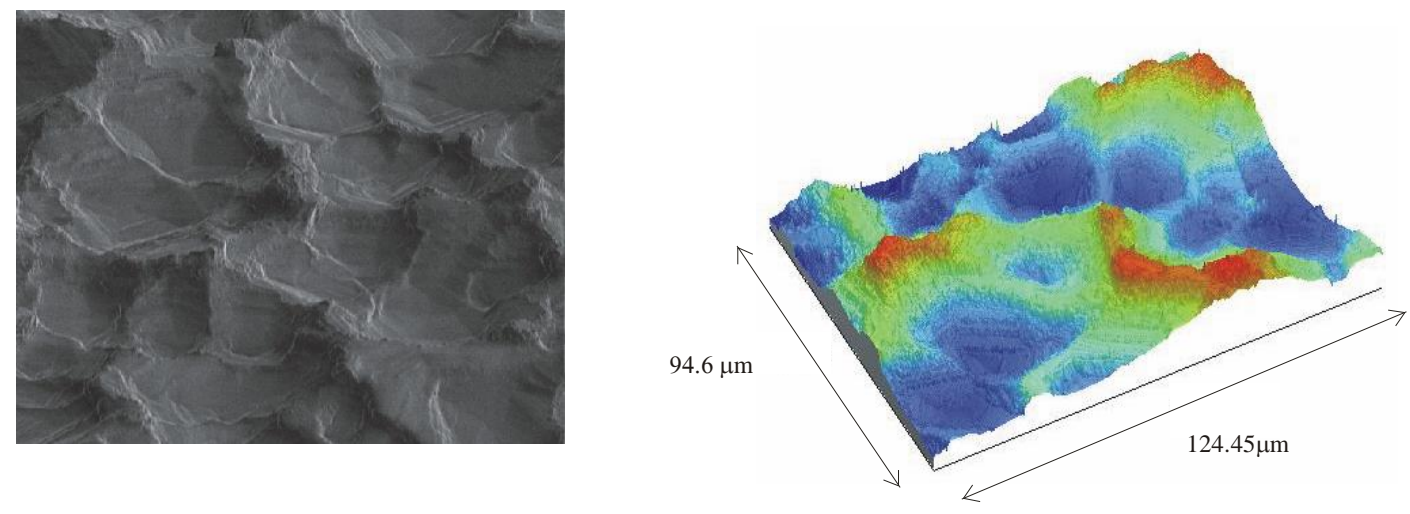

b)

Figure 3 


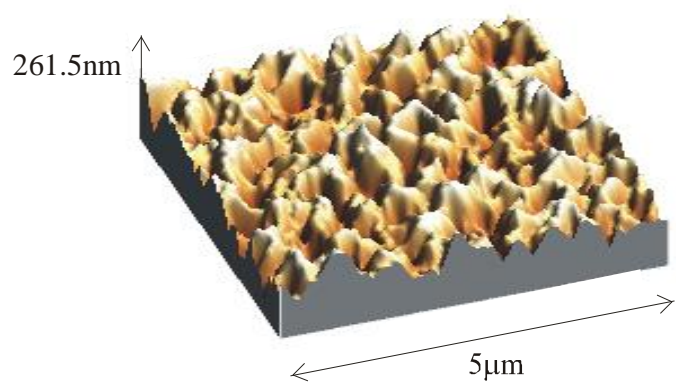

a)

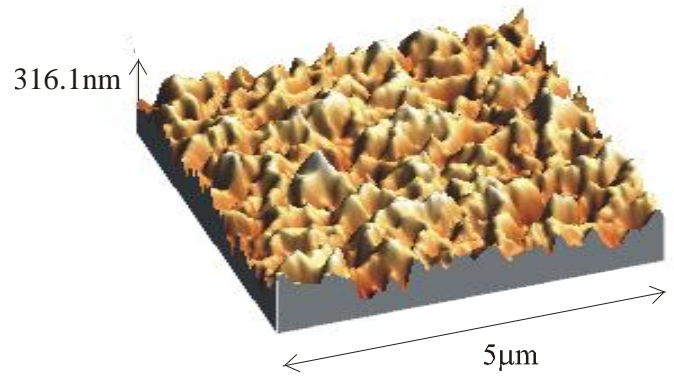

b)

Figure 4 


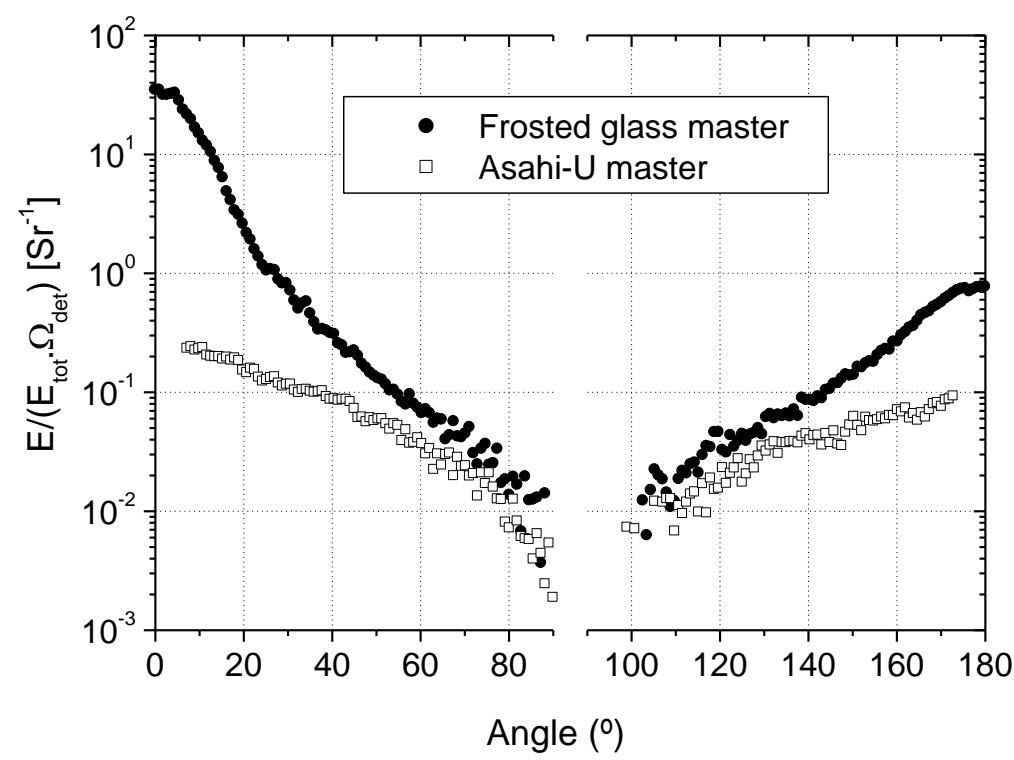

Figure 5 


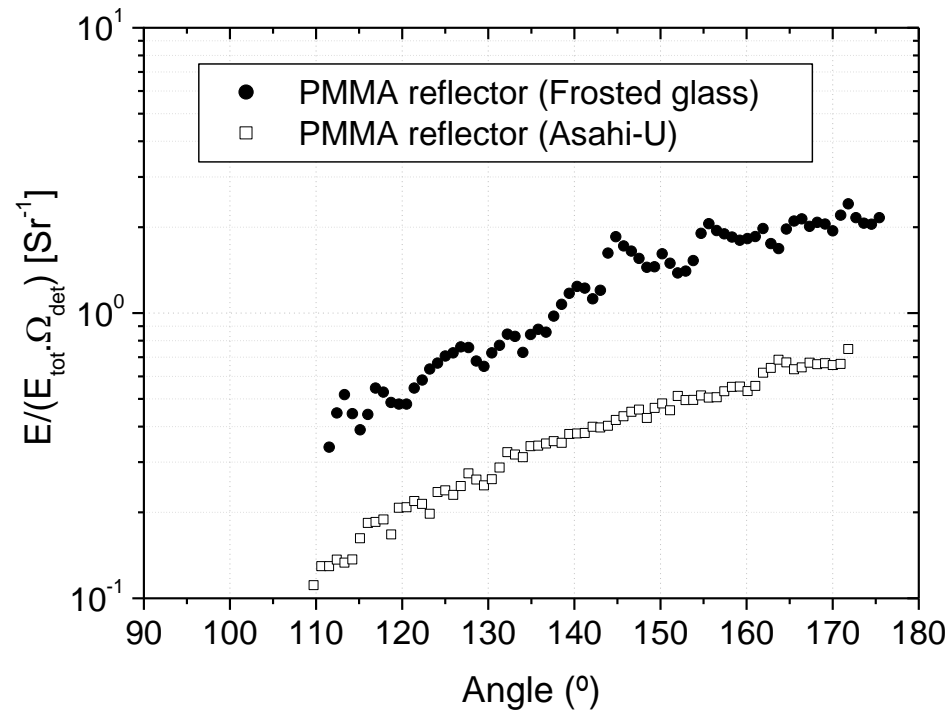

Figure 6 